\title{
Torsion of a Normal Ovary During the Early Postpartum Period
}

\author{
Yusuke Tanaka, MD, Shinsuke Koyama, MD, PhD, Yasuhiko Shiki, MD \\ Department of Obstetrics and Gynecology, Osaka Rosai Hospital, Osaka, Japan (all authors).
}

\begin{abstract}
Ovarian cyst torsion is a well-known cause of acute abdominal pain in female patients. However, torsion of a normal ovary is extremely rare and predominantly occurs in premenarchal female patients. The objective of this report is to describe a rare case of acute abdomen during the early postpartum period that was ultimately diagnosed as torsion of a normal ovary by laparoscopic surgery. A 29-year-old woman vaginally delivered a healthy 3530-g baby uneventfully at 40 weeks' gestation. On postpartum day 6 , she presented with acute abdominal pain in the right lower quadrant and costovertebral angle tenderness. A complete workup including laboratory examination, ultrasonography, and computed tomography was performed. Leukocytosis with a left shift and a slightly enlarged right ovary were found. Finally, diagnostic laparoscopy showed torsion of a normal ovary. The patient underwent laparoscopic detorsion of the right adnexa and was discharged on postoperative day 4 without any complications.
\end{abstract}

Key Words: Adnexal torsion, Acute abdomen, Laparoscopy, Postpartum, Pregnancy.

Citation Torsion of a normal ovary during the early postpartum period. Tanaka Y, Koyama S, Shiki Y. CRSLS e2013.00250. DOI: 10.4293/CRSLS.2013.00250.

Copyright $\odot 2015$ by SLS, Society of Laparoendoscopic Surgeons. This is an open-access article distributed under the terms of the Creative Commons Attribution-Noncommercial-ShareAlike 3.0 Unported license, which permits unrestricted noncommercial use, distribution, and reproduction in any medium, provided the original author and source are credited.

Address correspondence to: Yusuke Tanaka, MD, Department of Obstetrics and Gynecology, Osaka Rosai Hospital, 1179-3 Nagasone-cho, Kita-ku, Sakai, Osaka 591-8025, Japan. Telephone: +81-72-252-3561, Fax: +81-72-255-3349, E-mail: osaka-banzai@road.ocn.ne.jp

\section{INTRODUCTION}

Diagnostic laparoscopy is a safe and well-tolerated procedure that can be performed in carefully selected patients with acute abdomen. Adnexal torsion is one of the indications for diagnostic laparoscopy and accounts for approximately $3 \%$ of gynecologic emergencies. ${ }^{1}$ Although adnexal torsion during pregnancy or the postpartum period is often confused with other common diseases, it should not be overlooked. A delay in the diagnosis of adnexal torsion can lead to irreversible ovarian necrosis.

We report a rare case of torsion of a normal ovary during the early postpartum period that was diagnosed and treated by laparoscopic surgery. We present the clinical course of our case and review the literature concerning adnexal torsion during pregnancy and the peripartum period.

\section{CASE DESCRIPTION}

A healthy 29-year-old nulliparous woman conceived spontaneously. She had a history of cervical cone biopsy for cervical intraepithelial neoplasia, grade 3. No adnexal masses were detected by ultrasonography during the first trimester. The clinical course of her pregnancy was uneventful. At 40 weeks' gestation, she vaginally delivered a healthy 3530-g baby uneventfully. On postpartum day 6 , the patient presented with acute abdominal pain. A physical examination showed rebound tenderness in the right lower quadrant and costovertebral angle tenderness. Her body temperature was $37.1^{\circ} \mathrm{C}$. Her systolic and diastolic blood pressure was $96 \mathrm{~mm} \mathrm{Hg}$ and $57 \mathrm{~mm} \mathrm{Hg}$, respectively. The blood profile was as follows: white blood cell count, $16100 / \mu \mathrm{L}$ with $87.2 \%$ neutrophils; hemoglobin level, $11.1 \mathrm{~g} / \mathrm{dL}$; and platelet count, $308 \times 10^{9} / \mathrm{L}$. The urinalysis findings were normal. Transabdominal and transvaginal ultrasonography did not detect any ovarian cysts. An appendix was not detected. A contrast-enhanced computed tomography (CT) scan showed that the right ovary was slightly enlarged without any mass; it measured approximately $4 \mathrm{~cm}$ in size (Figure 1). There was no evidence of appendicitis or septic thrombophlebitis (ovarian vein thrombosis). We performed diagnostic laparoscopy, and the intraoperative findings confirmed that a normal ovary on the right side was twisted with no find- 


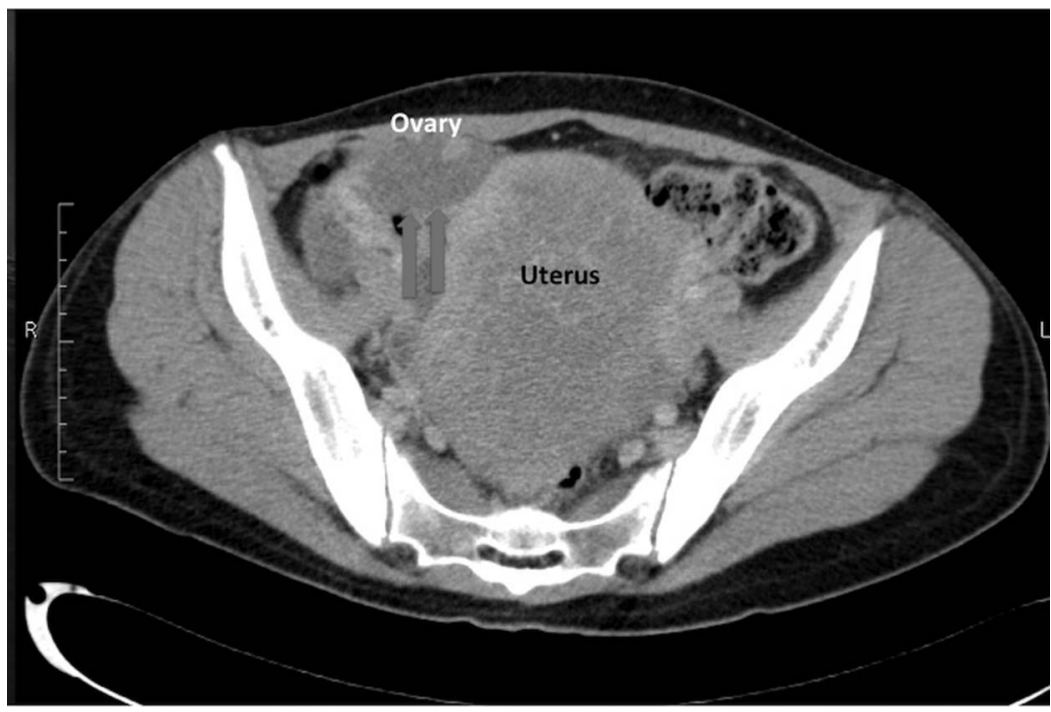

Figure 1. The right ovary was slightly enlarged and located anterior to the uterus (arrow).

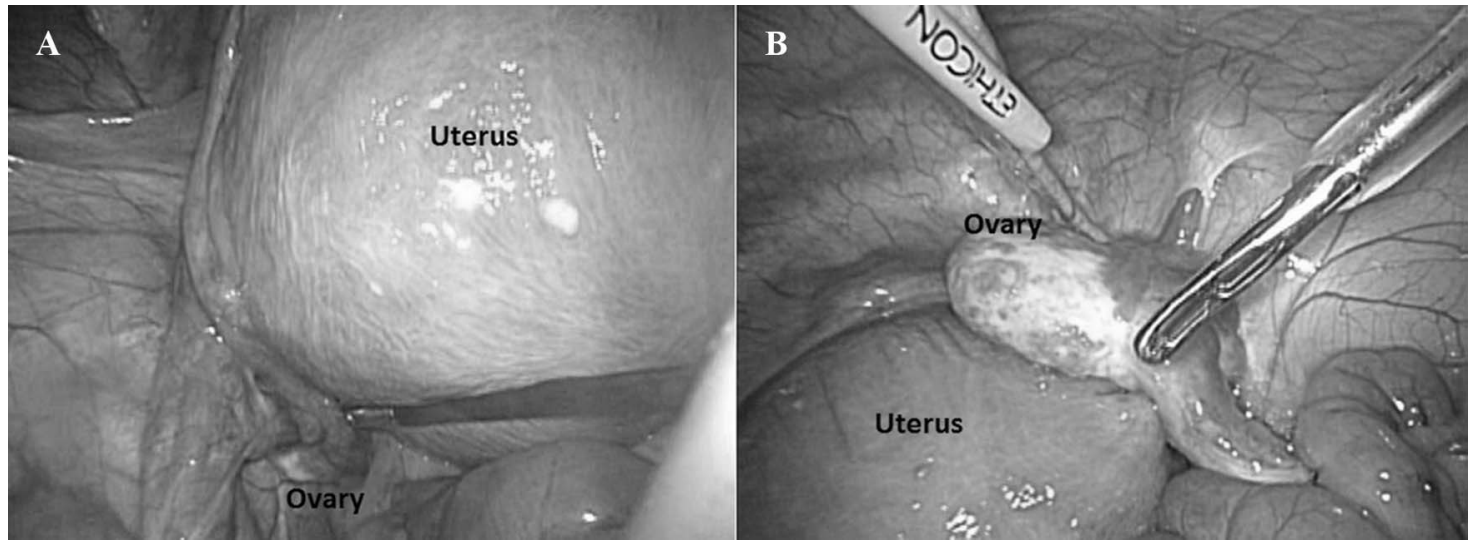

Figure 2. A, The left ovary was in its normal position. B, Diagnostic laparoscopy confirmed the presence of torsion of a normal ovary. The right ovary was twisted and located anterior to the uterus.

ings of severe necrosis (Figure 2). Therefore laparoscopic detorsion of the twisted adnexa was performed. The total length of the operation was 61 minutes, and the amount of estimated blood loss was $<50 \mathrm{~mL}$. The patient made a good recovery and was discharged on postpartum day 10 . She has been doing well during the postoperative follow-up period.

\section{DISCUSSION}

The most common symptoms of adnexal torsion are acute and severe abdominal pain, flank pain, fever, nausea, and vomiting. ${ }^{2}$ During pregnancy or the postpartum period, a diagnostic dilemma occurs because the clinical symptoms and laboratory findings are nonspecific and thus can be easily confused with other diagnostic possibilities including adnexal abscess, endometritis, postpartum ovarian vein thrombosis, retroperitoneal hematoma, acute appendicitis, intestinal obstruction, urinary calculus, and urinary tract infection. If adnexal torsion is suspected, surgery should not be delayed because the viability of the ovary may be compromised.

In women presenting with acute abdominal pain, bowel or urologic causes are considered common and will often lead to CT or magnetic resonance imaging as the first imaging study. The most common but nonspecific finding of ovarian torsion on CT is an enlarged ovary $(>4 \mathrm{~cm}$ in maximal dimension) with or without a mass. ${ }^{3}$ The enlarged ovary with a central afollicular stroma results from 
edema and hemorrhage. The extent of hemorrhage is dependent on the degree and duration of torsion, with hemorrhagic infarction occurring at a later stage than edema. ${ }^{4}$ Hiller et $\mathrm{al}^{5}$ reported that the correct preoperative diagnosis of adnexal torsion based on CT findings was made in only $34 \%$ of patients. As a result, the diagnostic accuracy of adnexal torsion based on CT findings seems to be low.

Doppler sonography has been considered a less invasive approach to detect ovarian torsion. Some authors have evaluated the rate of a correct diagnosis of ovarian torsion and identified any correlations with Doppler sonography. A recent study noted that a preoperative diagnosis was confirmed in only $46.1 \%$ of patients. ${ }^{6}$ The accuracy of the preoperative diagnosis of ovarian torsion remains low. Other authors have warned against the presence of a "normal flow on Doppler sonography." Their study noted that torsion was missed in $60 \%$ of cases with normal Doppler flow. ${ }^{7}$ The detection of normal flow by Doppler sonography does not exclude ovarian torsion. Despite great progress in diagnostic accuracy with advanced imaging by Doppler sonography, CT, and magnetic resonance imaging, many conditions require diagnostic laparoscopy to make an accurate diagnosis of acute abdomen.

Currently, diagnostic laparoscopy is technically feasible and can be applied safely in appropriately selected patients with acute abdomen. This technique not only facilitates the diagnosis of intra-abdominal disease but also makes therapeutic intervention possible. ${ }^{8}$ Although a high diagnostic accuracy (70\%-99\%) has been shown according to a review of the literature by Stefanidis et al, 9 the possibility of unexpected causes for acute abdomen that require additional therapeutic procedures should not be ruled out. Therefore it is important for gynecologists who are going to perform diagnostic laparoscopy to consult other specialists for assistance in managing this condition in advance.

The incidence of ovarian masses during pregnancy varies from approximately 1 in 100 to 1 in 2000 pregnancies. ${ }^{10}$ Adnexal torsion occurs in $3 \%$ of pregnant female patients with ovarian cysts. ${ }^{11}$ The selection of the management (conservative or surgical) depends on the size of the lesion and the ultrasonographic appearance. It seems reasonable to remove all ovarian masses $>10 \mathrm{~cm}$ because of the risk of torsion and the substantive risk of malignancy. ${ }^{10}$ Adnexal torsion in these cases may occur at any time during gestation, although its incidence decreases as the gestational age increases. ${ }^{12-14}$ In contrast, torsion of the normal adnexa during pregnancy or the postpartum period is uncommon, and the true incidence of such a rare condition is unclear. According to the previously reported literature, torsion of a normal ovary predominantly occurs in premenarchal female patients, in whom torsion involving previously normal adnexa may constitute up to $15 \%$ to $50 \%$ of adnexal torsion cases. ${ }^{15-17}$

The etiology of torsion of the normal adnexa is also not well defined. In the pediatric population, several authors have made speculations on this rare condition. ${ }^{15,16}$ The uterus is relatively small and the utero-ovarian ligaments are disproportionately long, which thus allows the ovary to twist easily. ${ }^{15}$ Other proposed mechanisms include abrupt changes in intra-abdominal pressure with vomiting and coughing, adnexal venous congestion due to perimenarchal hormone activity, and sudden acceleration/deceleration movements. ${ }^{16}$ We propose a possible explanation for the occurrence of torsion in an otherwise-normal ovary in the postpartum period: The puerperal patient might be more prone to the development of adnexal torsion because of the rapid anatomic changes in the pelvis, accompanied by the involution of the uterus while the utero-ovarian ligament remains disproportionately stretched, thereby allowing the normal-sized ovary increased room to move and twist.

In conclusion, torsion of a normal ovary during the postpartum period is an extremely rare but serious complication. The differential diagnoses of acute abdomen after delivery range widely. Physicians should recognize the importance of avoiding a delay in the diagnosis and treatment of ovarian torsion.

\section{References:}

1. Hibbard LT. Adnexal torsion. Am J Obstet Gynecol. 1985;152: $456-461$.

2. Ginath S, Shalev A, Keidar R, et al. Differences between adnexal torsion in pregnant and nonpregnant women. J Minim Invasive Gynecol. 2012;19:708-714.

3. Duigenan S, Pliva E, Lee SI. Ovarian torsion: diagnostic features on CT and MRI with pathologic correlation. AJR Am J Roentgenol. 2012;198:W122-W131.

4. Kawakami K, Murata K, Kawaguchi N, et al. Hemorrhagic infarction of the diseased ovary: a common MR finding in two cases. Magn Reson Imaging. 1993;11:595-597.

5. Hiller N, Appelbaum L, Simanovsky N, Lev-Sagi A, Aharoni D, Sella T. CT features of adnexal torsion. AJR Am J Roentgenol. 2007;189:124-129. 
6. Bar-On S, Mashiach R, Stockheim D, et al. Emergency laparoscopy for suspected ovarian torsion: are we too hasty to operate? Fertil Steril. 2010;93:2012-2015.

7. Peña JE, Ufberg D, Cooney N, Denis AL. Usefulness of Doppler sonography in the diagnosis of ovarian torsion. Fertil Steril. 2000;73:1047-1050.

8. Hori Y, SAGES Guidelines Committee. Diagnostic laparoscopy guidelines. Surg Endosc. 2008;22:1353-1383.

9. Stefanidis D, Richardson WS, Chang L, Earle DB, Fanelli RD. The role of diagnostic laparoscopy for acute abdominal conditions: an evidence-based review. Surg Endosc. 2009;23:16-23.

10. Cunningham FG, Leveno KJ, Bloom SL, Hauth JC, Rouse DJ, Spong CY. Reproductive tract abnormalities. In: Cunningham FG, Leveno KJ, Bloom SL, Hauth JC, Rouse DJ, Spong CY, eds. Williams Obstetrics. 23rd ed. New York, NY: McGraw-Hill; 2010:890-911.

11. Condous G, Khalid A, Okaro E, Bourne T. Should we be examining the ovaries in pregnancy? Prevalence and natural history of adnexal pathology detected at first-trimester sonography. Ultrasound Obstet Gynecol. 2004;24:62-66.
12. Rackow BW, Patrizio P. Successful pregnancy complicated by early and late adnexal torsion after in vitro fertilization. Fertil Steril. 2007;87:697.e9-697.e12.

13. Pansky M, Feingold M, Maymon R, Ami IB, Halperin R, Smorgick N. Maternal adnexal torsion in pregnancy is associated with significant risk of recurrence. J Minim Invasive Gynecol. 2009;16:551-553.

14. Hasson J, Tsafrir Z, Azem F, et al. Comparison of adnexal torsion between pregnant and nonpregnant women. Am J Obstet Gynecol. 2010;202:536.e1-536.e6.

15. Kokoska ER, Keller MS, Weber TR. Acute ovarian torsion in children. Am J Surg. 2000;180:462-465.

16. Mordehai J, Mares AJ, Barki Y, Finaly R, Meizner I. Torsion of uterine adnexa in neonates and children: a report of 20 cases. J Pediatr Surg. 1991;26:1195-1199.

17. Pansky M, Abargil A, Dreazen E, Golan A, Bukovsky I, Herman A. Conservative management of adnexal torsion in premenarchal girls. J Am Assoc Gynecol Laparosc. 2000;7:121124. 